who showed T2H ( $\mathrm{n}=58$ ) had significantly depressed verbal, performance, and full-scale IQ scores. On a test of fine motor skills, patients with T2H on MRI showed lower scores than those with normal MRI. Cognitive and motor performances of patients with T2H were not significantly correlated with the number of cerebral regions affected by $\mathrm{T} 2 \mathrm{H}$. Foci of $\mathrm{T} 2 \mathrm{H}$ affected a single cerebral region in $40 \%$ of patients with $\mathrm{T} 2 \mathrm{H}, 2$ or 3 regions in $48 \%$, and $12 \%$ had 4 or more regions affected. Hyperintensities on T2-weighted MRI represent a biological marker for impaired cognitive and fine motor performance in patients with NF1. (Feldmann R, Denecke J, Grenzebach $M$ et al. Neurofibromatosis type 1. Motor and cognitive function and T2-weighted MRI intensities. Neurology December (2 of 2) 2003;61:1725-1728). (Reprints: Dr R Feldmann, Department of Pediatrics, University of Munster, Albert-Schweitzer St, 33, D-48129 Munster, Germany).

COMMENT. This study demonstrates a strong relation between cognitive and fine motor performance and the presence of T2 hyperintensities on the MRI of patients with NF1. The number of cerebral regions affected by $\mathrm{T} 2 \mathrm{H}$ is not correlated with cognitive and motor performances. Previous reports have shown variable results. In a study of 12 families reported from Johns Hopkins Hospital, Baltimore, children with NF1 compared to unaffected siblings had a lower !Q, multifocal cognitive deficits, and reading and neuromotor disabilities. In this study, cognitive differences correlated with the number of MRI "unidentified bright objects" (T2H). (Hofman KJ, Denckla MB et al. J Pediatr 1994;124:S1S8). Patients identified with $\mathrm{T} 2 \mathrm{H}$ should be considered for special education services.

\title{
FUNCTION OF TUBERIN AND HAMARTIN IN TUBEROUS SCLEROSIS PATHOGENESIS
}

The genetics and function of tuberin and hamartin in the pathogenesis of tuberous sclerosis complex (TS) are reviewed in a presentation at a "Festschrift" honoring Dr Michael J Painter of the Division of Pediatric Neurology, Children's Hospital of Pittsburgh, PA. The prevalence of TS is $1 / 10,000$, two-thirds being sporadic. Among familial cases, half are linked to chromosome 9q34 (TSC1) locus and half to the 16p13.3 (TSC2) locus. Most sporadic cases are due to defects in TSC2. The TSC1 gene encodes the protein, hamartin, and the TSC2 gene encodes tuberin. The gene for polycystic kidney disease is located centromeric to TSC2, accounting for the occurrence of both conditions in families with contiguous gene syndromes. Tuberin and hamartin function as tumor suppressors, inhibiting the activity of rapamycin (mTOR) and regulating cell growth. (Narayanan V. Tuberous sclerosis complex: genetics to pathogenesis. Pediatr Neurol Nov 2003;29:404-409). (Respond: Dr Narayanan, Barrow Neurological Institute, St Joseph's Hospital and Medical Center, 500 W Thomas Rd, Suite 930, Phoenix, AZ 85013).

COMMENT. It is suggested that knowledge of the cellular functions of tuberin and hamartin might lead to new drugs that modulate signaling pathways. These could be effective in the control of epilepsy and cognitive impairment associated with TS. The discovery of tumor suppressor genes has provided insight into genetic alterations that contribute to neoplasia. These genes contribute to tumorigenesis only when both alleles have been inactivated. (Charrow J. In: Progress in Pediatric Neurology III, PNB Publ, 1997; Chpt 10:435-439). 